\title{
Surgical shortcuts with comments
}

\section{The importance of avoiding a laparotomy in case of hysterectomy}

Muzii L, Basile S, Zupi E, Marconi D, Zullo MA, Manci N, Bellati F, Angioli R, Benedetti Panici P.

Laparoscopic-assisted vaginal hysterectomy versus minilaparatomy hysterectomy: a prospective, randomized, multicenter study. J Minim Invasive Gynecol. 2007; 14: 610-5.

Study objective: The aim of this study was to compare operative and early postoperative outcomes of laparoscopic-assisted vaginal hysterectomy (LAVH) and minilaparotomy in a randomized clinical trial including patients undergoing total hysterectomy for benign gynecologic disease and having one or more of the generally considered contraindications to vaginal route. Design: Prospective, randomized, multicenter trial (Canadian Task Force Classification I). Setting: Departments of Gynecology from three major university hospitals in Rome. Patients: Eighty-one patients who were candidates for abdominal hysterectomy. Interventions: Laparoscopic-assisted vaginal hysterectomy and minilaparotomy hysterectomy. Measurements and main results: Forty patients were randomized to LAVH and 41 to minilaparotomy. The characteristics of the patients and the indications for surgery in the two arms were comparable. In the minilaparotomy group, complications were as follows: one case $(2.4 \%)$ of delayed laparotomy with $2 \mathrm{U}$ of red blood cell transfusion, two cases $(4.8 \%)$ of wound infection, and three cases $(7.3 \%)$ of fever of unknown origin. No minor or major complications were observed in the LAVH group. Postoperative visual analog scale pain scores at days 1 and 2 were significantly lower in the LAVH group $(p<.05)$. The complication rate between the two groups was significantly lower for LAVH $(p=.026)$. Conclusion: Because LAVH was associated with significantly lower early postoperative pain scores and complication rates, in general, LAVH should be preferred to minilaparotomy hysterectomy when the vaginal approach cannot be used.

\section{COMMENTARY}

The article describes a comparative study between laparoscopic-assisted vaginal hysterectomy (LAVH) and hysterectomy by minilaparotomy (TAH). Patients who had an estimated uterine size greater than $280 \mathrm{~g}$, adhesions due to previous pelvic surgery, nulliparity, adnexal disease, moderate or severe endometriosis were included in the study. Exclusion criteria were uterine size greater than $700 \mathrm{~g}$, previous midline laparotomy and absolute contraindications to laparoscopy. The authors conclude that the LAVH has a significantly longer operative time but lower postoperative pain and complication rates and should be preferred to the TAH by minilaparotomy. This recommended article demonstrates, as several quoted studies in the article, the importance of avoiding a laparotomy in case of hysterectomy. Although the 81 patients were recruited and operated in three different surgical units, statistical significance could be obtained. The authors acknowledge the mean goal in hysterectomy, to use the vaginal or laparoscopic route whenever possible. As a next step, it would be interesting to compare these data with the total laparoscopic hysterectomy (TLH).

This study is an important confirmation of the gold standard fixed during several minimal invasive consensus meetings.

\section{Rudy Leon De Wilde}

\section{The innate ability for dexterity and ambidexterity differs across handedness and gender}

\section{Elneel FH, Carter F, Tang B, Cuschieri A.}

Extent of innate dexterity and ambidexterity across handedness and gender: Implications for training in laparoscopic surgery. Surg Endosc. 2008; 22: 31-7.

Background: As innate dexterity is considered one of the important predictors of eventual operative competence, an experimental human factors study was conducted to determine innate dexterity and ambidexterity across handedness and gender. Methods: Fifty medical students (right-handed males, left-handed males, and right-handed females) were recruited as participants in a study designed to assess innate dexterity and degree of ambidexterity for endoscopic manipulations in a validated virtual-reality simulator. All participants performed unilateral and bilateral tasks with both dominant and non-dominant hands in random sequence. The outcome measures were execution time, extent of ambidexterity (ambidexterity index), aiming errors, and maximum tissue damage. Results: Right-handed males 
exhibited a greater level of ambidexterity than left-handed males ( $p=0.02$ for path length, $p=0.001$ for angular path) and right-handed females ( $p=0.01$ for path length, $p=0.02$ for angular path), and more efficient task performance as measured by execution time $(p=0.001$ for males and $p=$ 0.03 across gender). The task quality when executed by the dominant hand was best in right-handed males $(p=0.001$ vs. left-dominant males and $p=0.03$ across gender). No significant difference was observed in terms of precision control and fine movements (aiming errors and maximum tissue damage) between the three groups. Conclusions: These findings indicate that training surgical curricula in laparoscopic surgery should be more flexible to accommodate the innate differences across handedness and gender.

\section{COMMENTARY}

The authors conducted a prospective study to evaluate the degree of innate dexterity and ambidexterity for laparoscopic skills, taking into account that innate dexterity is one of the predictors of eventual surgical competence.

The study was performed on 50 medical students to be able to determine innate abilities prior to any surgical training. The effect of handedness was evaluated by comparing the results of right-handed males with those of left-handed males, whereas the effect of gender was evaluated by comparing the results of right-handed males with those of right-handed females.

Participants performed standardised unimanual task (navigation) and bimanual task (lifting and grasping) with both the dominant and non-dominant hands in random order in a validated virtual-reality simulator (LapSim Basic Skills). The outcomes measures were execution time, aiming errors and tissue damage for both tasks and also the ambidexterity index (difference between dominant hand and non-dominant hand) for the unimanual task.

For the unimanual task, right-handed males had a greater degree of ambidexterity than left-handed males and than right-handed females. The performance by the dominant hand of right-handed males was better than that achieved by the dominant hand of left-handed males and of righthanded females. The performance by the non-dominant hand of the three groups was comparable. No difference in terms of precision control and fine movements (aiming errors and tissue damage) was detected between the three groups.

For the bimanual task, right-handed males had a shorter execution time than left-handed males and than righthanded females without differences for aiming errors and tissue damage.

The data is consistent with previous reports confirming that right-handed males have better performance. However, some limitations should be considered at interpreting the results, as it is very nicely discussed by the authors. Indeed, the instrument handles used as the interface with the virtual-reality simulator were similar to the conventional instruments used in laparoscopic surgery, designed for use by right-handed individuals and on the principle of one size fits all. This implies some obvious disadvantages for the left-handed males and the females due to their smaller hands. Furthermore, no data are available about left-handed females.

This article may have important implications for training in laparoscopic surgery as it demonstrates that the innate ability for dexterity and ambidexterity differs across handedness and gender. It is, therefore, strongly recommended for those involved in programming curricula for training in laparoscopy. The study suggest that the fixed curriculum, either for the laboratory or for the operating theatre, should be revised and adapted to the trainee's requirements to be able to counteract these innate differences and to eventually achieve surgical proficiency. The article provides an important confirmation that right-handed males have greater skills and open new insights into the factors influencing the acquisition of laparoscopic psychomotor skills.

\section{Rudi Campo}

The size of the differences in costs and quality of life between uterine artery embolisation and hysterectomy is small

Wu O, Briggs A, Dutton S, Hirst A, Maresh M, Nicholson A, McPherson K.

Uterine artery embolisation or hysterectomy for the treatment of symptomatic uterine fibroids: a cost-utility analysis of the HOPEFUL study. BJOG. 2007; 114: 1352-62.

Objectives: To evaluate the relative cost-effectiveness of uterine artery embolisation (UAE) and hysterectomy in women with symptomatic uterine fibroids from the perspective of the UK NHS. Design: Cost-utility analysis. Setting: Eighteen UK NHS hospital trusts. Population or sample: Women who underwent $\operatorname{UAE}(n=649$; average follow-up of 8.6 years) or hysterectomy ( $n=459$; average follow-up of 4.6 years) for the treatments of symptomatic fibroids. Methods: A probabilistic decision model was carried out based on data from a large comparative cohort and the literature. The two interventions were evaluated over the time horizon from the initial procedure to menopause. Extensive sensitivity analysis was carried out to test model assumptions and parameter uncertainties. Main outcome measures: Costs of procedures and complications and quality of life expressed as quality-adjusted life years (QALYs). Results: Overall, UAE was associated with lower mean cost (2,536 versus 3,282 pounds sterling) and a 
small reduction in quality of life (8.203 versus 8.241 QALYs) when compared with hysterectomy. However, when the quality of life associated with the conservation of the uterus was incorporated in the model, UAE was shown to be the dominant strategy-lower costs and greater QALYs. Conclusions: UAE is a less-expensive option to the health service compared with hysterectomy, even when the costs of repeat procedures and associated complications are factored in. The quality of life implications in the shortterm are also predicted to favour UAE; however, this advantage may be eroded over time as women undergo additional procedures to deal with recurrent fibroids. Given that hysterectomy is the current standard treatment for symptomatic fibroids, offering women UAE as an alternative treatment for fibroids is likely to be highly cost-effective for those women who prefer uterus-conserving treatment.

\section{COMMENTARY}

Uterine artery embolisation (UAE) is a new technique that provides an alternative to myomectomy or hysterectomy in patients with symptomatic uterine fibroids. As many of the patients having had an UAE have been followed-up for only a short time and, in some UAE cases, a repeat procedure has to be performed, the long-term complication rate and cost effectiveness of UAE is still unclear. In the recently reported EMMY trial from The Netherlands, Volkers et al. reported that $23.5 \%$ of the patients randomized to embolization subsequently underwent hysterectomy during the first 2 years of follow-up (Am J Obstet Gynecol 2007;196:519). Therefore, it is important to continue to evaluate UAE, especially in the long-term.

The article by $\mathrm{Wu}$ et al. is a companion work of the HOPEFUL study, a UK multicenter retrospective cohort study comparing hysterectomy and UAE for the treatment of symptomatic uterine fibroids. The results of the HOPEFUL study on medium-term safety and efficacy have been published (BJOG 2007;114:1340-1351). In the present study, $\mathrm{Wu}$ et al. analyzed the cost utility of UAE compared with hysterectomy. The average follow-up was 8.6 years for the 649 women treated by UAE and the average follow-up was 4.6 years for the 459 women who underwent a hysterectomy. The costs of procedures and complications and the quality of life expressed as quality-adjusted life years (QALYs) were evaluated for both procedures.

The cost-utility analysis was based on data from the HOPEFUL cohort, a multicenter retrospective cohort of women who received UAE or hysterectomy. The cost model presented by $\mathrm{Wu}$ et al. is based on a retrospective questionnaire, and the literature and might differ from the real costs. Complications from UAE and from hysterectomy were included in the cost analysis. However, the authors decided to exclude symptoms of "postembolization syndrome" from the complication list. Including these complications would have increased the costs generated by UAE. Furthermore, the hysterectomy group included all types of hysterectomy. As the size of the differences in costs and quality of life between UAE and hysterectomy was small, it would have been interesting to compare the UAE costs to costs associated to laparoscopic hysterectomy. Laparoscopic hysterectomy is related with lower costs associated with loss of productivity.

This interesting article shows that the size of the differences in costs and quality of life between UAE and hysterectomy is small. Overall, the balance of whether UAE improves quality of life is likely to rest with the woman and her attitudes towards the resolution of fibroid symptoms and the preservation of her uterus. Therefore, it would also be very important to compare UAE to laparoscopic myomectomy.

Michael D. Mueller 